\title{
Cortical presentation of language functions in patients after total laryngectomy: a fMRI study
}

\author{
Aleksandra Wypych $^{1}$ - Małgorzata Wierzchowska ${ }^{2}$ - Paweł Burduk ${ }^{2}$ Elżbieta Zawada ${ }^{3,4} \cdot$ Katarzyna Nadolska $^{3}$. \\ Zbigniew Serafin ${ }^{3}$
}

Received: 4 December 2019 / Accepted: 13 March 2020 / Published online: 6 April 2020

(C) The Author(s) 2020

\begin{abstract}
Purpose The aim of this study is to use functional magnetic resonance (fMRI) to analyse the cortical presentation of selected language functions in patients after a total laryngectomy.

Methods Eighteen patients after total laryngectomy treated with electrolarynx speech and 18 volunteers were included. The mean number of patients' post-operative speech rehabilitation sessions was five (range of 3-8 sessions). Four paradigms were used, including noun generation, pseudoword reading, reading phrases with pseudowords, and nonliteral sign reproduction.

Results In noun, the most significant difference between the groups was the stronger activation of both lingual gyri in the volunteers. Pseudoword reading resulted in stronger activations in patients than in volunteers in the lingual gyri, the right cerebellum, the right Broca's area, and the right parietal operculum. Reading phrases with pseudowords involved different parts of the Brodmann area 40. During nonliteral sign reproduction, there was a stronger activation of the left Broca's area in volunteers and a stronger activation of the left premotor cortex in patients.

Conclusion This study provides evidence of altered cortical activation in response to language tasks in patients after a laryngectomy compared with healthy volunteers, which may be considered brain plasticity in response to a laryngectomy.
\end{abstract}

Keywords Laryngectomy $\cdot$ Magnetic resonance imaging $\cdot$ Gray matter

\section{Introduction}

The ability to speak is one of the sophisticated features that make a distinction between humans and other animals. No wonder that how the brain controls speech has remained a subject of investigation for centuries. The pioneers of research on cerebral language functions were Pierre Paul Broca and Karl Wernicke $[1,2]$. Their studies and theories had a

Zbigniew Serafin

serafin@cm.umk.pl

1 The Interdisciplinary Center for Modern Technologies, Nicolaus Copernicus University, Toruń, Poland

2 Department of Otolaryngology, Oncology and Oral and Maxillofacial Surgery, Nicolaus Copernicus University, Collegium Medicum, Bydgoszcz, Poland

3 Department of Radiology and Diagnostic Imaging, Nicolaus Copernicus University, Collegium Medicum, Bydgoszcz, Poland

4 Department of Geriatrics, Nicolaus Copernicus University, Collegium Medicum, Bydgoszcz, Poland profound effect on contemporary understanding of speech. Sakai et al. [3] commented that human language is a unique faculty of the mind. The grammaticality of a sentence, they assert, needs to be made explicit by the adoption of an appropriate theoretical framework for linguistic structures. Grammatical rules arise from the human brain so that language must be considered a subsystem of the mind, with the language system being a distinct module, which in turn possesses its own modularity or subsystems such as phonology, semantics, and syntax, which interact systematically with each other through the information flow between them [3].

However, despite the huge progress in neuroimaging, the definite location of these areas still remains controversial [4, 5]. Some authors define it as a unimodal auditory association in the superior temporal gyrus anterior to the primary auditory cortex (the anterior part of BA 22) [6]. This is the site most consistently implicated in auditory word recognition by functional brain imaging experiments [7]. Others also include adjacent parts of the heteromodal cortex in Broca's area (BA) 39 and BA40 in the parietal lobe [8]. Recently, Ardila et al. suggested that grammar correlates with the ability to internally represent actions (verbs) depending on the functioning of BAs 
44 and 45 and the brain circuits related to them [9]. Grammar is also thought to be associated with the ability to quickly carry out the sequencing of the articulatory movements required for speaking (speech praxis) [10]. Meta-analytic studies which aimed to analyse the specific contribution of different BAs to the language system identified some areas potentially related to the adoption and comprehension of language (the lexical and semantic system) and areas related to language production (the grammatical system) $[9,11]$.

Total laryngectomy is still the method of choice for the treatment of advanced laryngeal cancer when radiotherapy fails to preserve the organ [12]. However, after a laryngectomy, patients not only lose the ability to communicate but also fall into a kind of social exclusion [12, 13]. Voice rehabilitation is a very important part of both pre-operative and postoperative treatment. The most technically advanced speech generation technique, ELS, uses an electronic device that generates sound regulated by vibrations of the patient's neck or cheek muscles [11]. It seems to be the most comparable to a healthy person's speech considering acoustic parameters including fundamental frequency, maximum phonation time, and intensity of the voice [11, 14-17].

Considering the complicated and not fully understood cortical control of speech, it is obvious that a sudden inability to speak in an adult, caused by a total laryngectomy, is a vast disablement to the patient. Moreover, speech rehabilitation methods, as described above, seem to stimulate cortical function remodelling. Remodelling of both connectivity and grey matter areas is a well-known phenomenon after brain injury and the loss of peripheral functions $[11,14]$. Several different language paradigms were proposed to localize speech-related brain areas $[18,19]$. Word generating is one of the most commonly used paradigms that results in activation mostly at the left inferior frontal gyrus and the bilateral motor cortex [20]. One may suppose that after laryngectomy, only the activation of motor cortex may be affected. Similarly, more or less sophisticated conceptual paradigms, including pseudoword reading and repeating, and nonliteral sign reproduction [21] cortical processing should not be influenced by laryngectomy as well.

We hypothesise that a similar process takes place after a laryngectomy. To our knowledge, there are no published studies that analyse brain cortical function related to a laryngectomy. The aim of this study was to use fMRI to analyse the cortical presentation of selected language functions in patients after a total laryngectomy.

\section{Methods}

\section{Participants}

The study group consisted of 36 right-handed subjects, including 18 patients after a total laryngectomy ( 15 men and 3 women at mean age $61 \pm 8$ years) and 18 healthy volunteers (15 men and 3 women at mean age $57 \pm 7$ years). All the patients were treated with ELS and were clinically considered subjects who successfully completed post-operative speech rehabilitation. The mean number of speech rehabilitation sessions was five (range of 3-8 sessions). Exclusion criteria were as follows: psychiatric or neurological disorders, previous brain or ear surgery, pregnancy, claustrophobia, metal foreign objects or metal implants (heart stimulator, cardioverter, insulin pump, cochlear implant, CNS stimulation, prosthesis). All participants were adults and gave informed consent to participate. We obtained permission to conduct the study from the local bioethical committee.

\section{Scanning and language tasks}

The study was conducted using a 3T MRI scanner (GE Discovery MR 750, General Electric Healthcare, Waukesha, IL, USA) and an 8-channel neurovascular head coil (HD 8CH Neurovascular Array, General Electric Healthcare, Waukesha, Il, USA). For each participant, scanning started with a high-resolution 3D BRAVO sequence (3D T1W1, TR $8.2 \mathrm{~ms}$, TE $3.2 \mathrm{~ms}$, FA $12^{\circ}$, thickness $1.0 \mathrm{~mm}$, no interslice gap, 176 slices, voxel size $1 \times 1 \times 1 \mathrm{~mm}$ ). Then, the functional experiment was performed using the BOLD technique in the axial plane using an EPI sequence (TR $2000 \mathrm{~ms}$, TE $28 \mathrm{~ms}$, FA $90^{\circ}$, thickness $3.0 \mathrm{~mm}$, no interslice gap, 44 slices, voxel size $3 \times 3 \times 3 \mathrm{~mm}$ ). In the fMRI experiment, four paradigms were used: noun generation, pseudoword reading, reading phrases with pseudowords, and nonliteral sign reproduction. The tasks were presented in the subjects' mother tongue (Polish) using NNL VisualSystem goggles (NordicNeuroLab AS, Bergen, Norway). Paradigms were created in Presentation (http://www.neurobs.com), a stimulus delivery and experiment control program for neuroscience.

Each language activation comprised one fMRI run per paradigm in block design experiments. Stimuli were presented as six $30 \mathrm{~s}$ active blocks separated by six $30 \mathrm{~s}$ rest pauses. Each session lasted $6 \mathrm{~min}, 10 \mathrm{~s}$, including 5 dummy scans. Tasks were always presented in the same order and were of the same duration. Participants were instructed before the MRI scanning about the structure of the study and they were given examples of tasks that were different from the paradigms presented in the scanner. For each task, the volunteers were asked only to think about a word:

In task 1 , they were asked to generate nouns beginning with a given letter (e.g. T, P, R, S, K).

In task 2, pseudonouns were presented in strings. The list of words was arranged gradually from the easiest to the most difficult in terms of structure (C means a consonant and $\mathrm{V}$ means a vowel): (i) two-syllable words with the CVC-CVC recording scheme, e.g. "chesnut"; (ii) 
polysyllable words consisting of a different number of syllables with a $\mathrm{CV}$ and $\mathrm{CVC}$ recording scheme, e.g. "chesstboard"; (iii) two-syllable words with a CCV-CV recording scheme, e.g. "prima". Each string was displayed for $30 \mathrm{~s}$ and consisted of five words. The subjects were instructed to read and repeat the pseudonouns in their minds until the next string of words appeared.

In task 3, participants were asked to read phrases consisting of single words with converted or shifted letters, e.g. "raed veihcle", "lietr of waeter".

In task 4, nonliteral sign reproduction (e.g. !,,+ , ,:) were presented. Participants were asked to repeat the name of graphic sign in their mind.

\section{Data processing and analysis}

Data pre-processing and analysis were performed using an FSL v. 5.0 (The FMRIB Software Library) toolkit. Images were controlled for field distortion, spike artefacts, and temporal signal-to-noise ratio. Then, EPI scans were subject to movement correction, co-registration, normalisation, segmentation, and spatial smoothing. Movement correction was conducted using a MCFLIRT package [10]. For structural and functional image registration, a FLIRT library (FMRIB's Linear Image Registration Tool) and the MNI152 standardspace T1-weighted average structural template image were used [9]. Spatial smoothing was performed with a Gaussian filter (FWHM $4.0 \mathrm{~mm}$ ). We also used a high-pass filter in a time domain (Gaussian-weighted least-squares straight line fitting, sigma $=50.0 \mathrm{~S}$ ). Time series analysis was conducted using FILM software (FMRIB's Improved Linear Model) with local correlation correction $[22,23]$. The fMRI data analysis was conducted using a FEAT library v. 6.00 (FMRI Expert Analysis Tool).

$\mathrm{Z}$ statistic images (Gaussianized $\mathrm{T} / \mathrm{F}$ ) were set in terms of clusters for $Z>2.3$. The cluster significance (with correction) was set at $p=0.05$. The first five EPI scans were discarded in each data package to achieve signal balance. Cluster correction was performed on both the first-level and the second-level analysis. The first-level statistical analysis (individual data) was conducted using a general linear model (GLM). The second-level statistical analysis (group analysis) for difference assessment between healthy controls and laryngectomy patients was conducted using a FLAME library (FMRIB's Local Analysis of Mixed Effects), which is a type of variance test for modelling and estimating the random-effects component of the measured inter-session mixed-effects variance in a full Bayesian network. FLAME 1 option with mixed-effects model was applied. Statistical analysis was made by the unpaired sample $t$ test. Each task was analysed in two variants: activation stronger in controls than in patients and activation stronger in patients than in controls. Significant clusters were labelled based on Juelich Histological Atlas [24].

\section{Results}

None of participants terminated the MRI examination and none of exams were rejected due to artefacts.

Results showed a stronger activation of the left visual cortex V3V and come part of the right visual cortex V2 in controls compared with patients during a noun generation task. Conversely, laryngectomy patients presented stronger activation of the right visual cortex $\mathrm{V} 1$, another part of the right visual cortex $\mathrm{V} 2$, the right inferior parietal lobule, the left cingulum, and the right premotor cortex (Table 1). On the other hand, in response to task 2, a stronger activation in volunteers was seen for visual cortex V3 and a stronger activation in patients for the right visual cortex $\mathrm{V} 2$, the left visual cortex V4, and the right Broca's area (Table 2).

Task 3 resulted in the strongest cortical activation in patients. The left Broca's area, the left anterior intra-parietal sulcus, and visual cortex (V3, V4) were strongly activated in patients, whereas V1 and V2 visual cortex, the left primary somatosensory cortex, and the left premotor cortex presented stronger response in controls (Table 3, Fig. 1). Finally, during nonliteral sign reproduction, controls activated more the left Broca area than patients, while patients activated more the left premotor cortex than controls (Table 4).

\section{Discussion}

To our knowledge, this is the first study published presenting differences between patients after a laryngectomy and healthy volunteers in cortical activation in response to language tasks.

Tasks resulted in activations at different levels of the visual cortex but no clear pattern could be defined in both volunteers and patients. The only difference was activation of the V3 visual cortex in volunteers in most of the experiments. Despite intensive investigation, the precise location and function of the third visual cortex remains a matter of debate [25, 26]. Generally, it is considered to play a role in the processing of motion, either global or coherent [25]. Based on the construction of tasks used in this study, the observed stronger activation of the V3 cortex in healthy subjects remains unclear.

Noun generation (task 1) requires semantic categorisation and, thus, makes great demands on semantic processing [27]. The most significant difference between the groups was the stronger activation of both lingual gyri in the volunteers. These areas are responsible for semantic categorisation, word retrieval, word generation, and single letter processing [28-31] so their activation presents a proper function. On 
Table 1 Response to task 1

\begin{tabular}{|c|c|c|c|c|c|}
\hline Juelich histological atlas & $Z$ value & $P$ value & $\mathrm{x}$ & $\mathrm{y}$ & $\mathrm{z}$ \\
\hline \multicolumn{6}{|l|}{ Activation stronger in healthy volunteers than in laryngectomy patients } \\
\hline Left visual cortex V3V & 2.86 & $<0.0000$ & 53 & 22 & 33 \\
\hline Right visual cortex V2 & 2.79 & 0.0002 & 41 & 22 & 33 \\
\hline Right visual cortex V3 & 2.53 & 0.0031 & 29 & 21 & 32 \\
\hline Right cingulum & 2.46 & 0.0042 & 42 & 40 & 49 \\
\hline Left secondary somatosensory cortex / parietal operculum OP4 & 2.43 & 0.0369 & 67 & 58 & 42 \\
\hline Visual cortex V1 & 2.43 & 0.0369 & 40 & 21 & 38 \\
\hline Right Broca's area & 2.40 & 0.0435 & 24 & 72 & 39 \\
\hline Right secondary somatosensory cortex/parietal operculum OP1 & 2.39 & 0.0435 & 18 & 50 & 47 \\
\hline \multicolumn{6}{|c|}{ Activation stronger activated in laryngectomy patients than in healthy volunteers } \\
\hline Right visual cortex V1 & 2.63 & 0.0002 & 37 & 17 & 38 \\
\hline Right visual cortex V2 & 2.61 & 0.0027 & 35 & 21 & 32 \\
\hline Right inferior parietal lobule Pga & 2.47 & 0.0028 & 18 & 35 & 52 \\
\hline Left cingulum & 2.42 & 0.0032 & 48 & 55 & 52 \\
\hline Right premotor cortex & 2.37 & 0.0036 & 37 & 55 & 67 \\
\hline Left premotor cortex & 2.32 & 0.0413 & 51 & 56 & 69 \\
\hline
\end{tabular}

the other hand, patients after a laryngectomy presented a stronger activation of the right angular gyrus, the left anterior cingulate gyrus, and the bilateral premotor cortex. The angular gyrus is surrounded by secondary somatosensory, visual and auditory cortical areas and is essential in the multimodal, highly complex synthesis of information [32]. Thus, it can be considered an adjuvant cortical area activated in patients. The anterior cingulum was linked to many different functions, including semantic and phonological verbal fluency [33]. However, in patients after a laryngectomy, activation of this area may be also linked to its role in cognitive and motor inhibition, motor imagery as well as in motor preparation and planning $[34,35]$. The premotor cortex, which is also connected with multiple functions, was activated for word generation in other studies [27]. Basic functions of this area include motor sequencing, movement planning, and imagination of movement [36, 37]. These functions may require stronger activation when oral speech has to be replaced by artificial phonation. The left premotor cortex is also responsible for speech initiation and speech motor programming [15, 38], which again require more effort after a laryngectomy.

Pseudoword reading (task 2 ) resulted in stronger activations in patients than in volunteers in the lingual gyri, the right cerebellum, the right Broca's area, and the right parietal operculum (OP1). Previous studies indicated that pseudoword reading involved the left fusiform gyrus, the left angular gyrus, and the left middle temporal gyrus for lexical and semantic processing. Furthermore, spelling-sound conversion was located in the left inferior parietal gyrus, and phonological output in the left inferior frontal gyrus [39]. Hauck et al. also found significant activation in the left inferior parietal gyrus, which confirms our results. In general, our results show more complicated processing of pseudoword reading after a laryngectomy. Activation of OP1 has been linked to literal sentence comprehension, and word imageability $[10,40]$. The contribution of the cerebellum to pseudoword processing is less clear. Guediche et al. postulated

Table 2 Response to task 2

\begin{tabular}{|c|c|c|c|c|c|}
\hline Juelich histological atlas & $Z$ value & $P$ value & $\mathrm{x}$ & $\mathrm{y}$ & $\mathrm{z}$ \\
\hline \multicolumn{6}{|l|}{ Activation stronger in healthy volunteers than in laryngectomy patients } \\
\hline Visual cortex V3 & 2.79 & 0.0042 & 34 & 23 & 32 \\
\hline \multicolumn{6}{|c|}{ Activation stronger activated in laryngectomy patients than in healthy volunteers } \\
\hline Right visual cortex V2 & 2.75 & $<0.0000$ & 38 & 23 & 33 \\
\hline Left visual cortex V4 & 2.55 & $<0.0000$ & 56 & 24 & 33 \\
\hline Right Broca's area & 2.45 & 0.0009 & 18 & 74 & 39 \\
\hline Right secondary somatosensory cortex/parietal operculum OP1 & 2.35 & 0.0019 & 17 & 51 & 47 \\
\hline Left visual cortex V1 & 2.33 & 0.0282 & 51 & 18 & 37 \\
\hline Left visual cortex V2 & 2.32 & 0.0313 & 49 & 16 & 33 \\
\hline
\end{tabular}


Table 3 Response to task 3

\begin{tabular}{lccccc}
\hline Juelich histological atlas & $Z$ value & $P$ value & $\mathrm{x}$ & $\mathrm{y}$ & $\mathrm{z}$ \\
\hline Activation stronger in healthy volunteers than & in laryngectomy patients \\
Left Broca's area & 3.10 & $<0.0000$ & 71 & 75 & 42 \\
Left anterior intra-parietal sulcus hip2 & 2.57 & $<0.0000$ & 67 & 44 & 54 \\
Right visual cortex V3 & 2.53 & $<0.0000$ & 34 & 23 & 32 \\
Right visual cortex V4 & 2.43 & 0.0006 & 29 & 25 & 31 \\
Left inferior parietal lobule pft & 2.39 & 0.0032 & 72 & 50 & 54 \\
Right visual cortex V2 & 2.32 & 0.0034 & 37 & 17 & 38 \\
Activation stronger activated in laryngectomy patients than in healthy \\
volunteers & & & & & \\
Left visual cortex V1 & 2.73 & 0.0010 & 49 & 15 & 37 \\
Right visual cortex V2 & 2.65 & 0.0002 & 37 & 21 & 32 \\
Left primary somatosensory cortex & 2.58 & 0.0035 & 71 & 55 & 58 \\
Left visual cortex V2 & 2.46 & 0.0028 & 49 & 16 & 33 \\
Left premotor cortex & 2.46 & 0.0020 & 51 & 56 & 69 \\
Right premotor cortex & 2.38 & 0.0252 & 38 & 55 & 71 \\
Left primary somatosensory cortex & 2.38 & 0.0392 & 70 & 52 & 60 \\
\hline
\end{tabular}

a functional network between the cerebellum and languagerelated regions in the temporal and parietal lobes contributing to sensorimotor adaptation. They stated that cerebro-cerebellar interactions may support supervised learning mechanisms that rely on sensory prediction error signals in speech perception [41]. Therefore, cerebellar activation in our patients may be a feature of brain plasticity in response to a laryngectomy.
Reading phrases with pseudowords (task 3 ) was a more complicated paradigm and involved different parts of the Brodmann area $40 \mathrm{~L}$ that is responsible for more elaborate semantic processing [42]. Apart from the activations discussed above, an interesting observation was a much stronger left Broca's area activation in volunteers than in patients, which again underlines altered speech processing after a laryngectomy. On the other hand, a stronger response from the left primary somatosencory cortex and the bilateral premotor cortex was observed in patients.

Cortical representation of nonliteral sign reproduction (task 4) remains a matter of debate. A meta-analysis by Rapp et al. indicated that a predominantly left lateralised network, including the left and right inferior frontal gyrus, the left, middle, and superior temporal gyrus, and medial prefrontal, superior frontal, cerebellar, parahippocampal, precentral, and inferior parietal regions, was important for nonliteral expressions [43]. On the other hand, Yang et al. concluded that there is flexible involvement of the sensory-motor system in abstract concept processing, which depends on semantic features of the language stimuli and links between abstract and literal meanings [12]. In our study, the most significant differences between the groups again included a stronger activation of the left Broca's area in volunteers and a stronger activation of the left premotor cortex in patients.

Some limitations of the current study have to be addressed. Firstly, the time of rehabilitation after a laryngectomy varied in our study group between three and eight learning sessions. We believe that the extent and the efficiency of speech processing plasticity in the brain may be

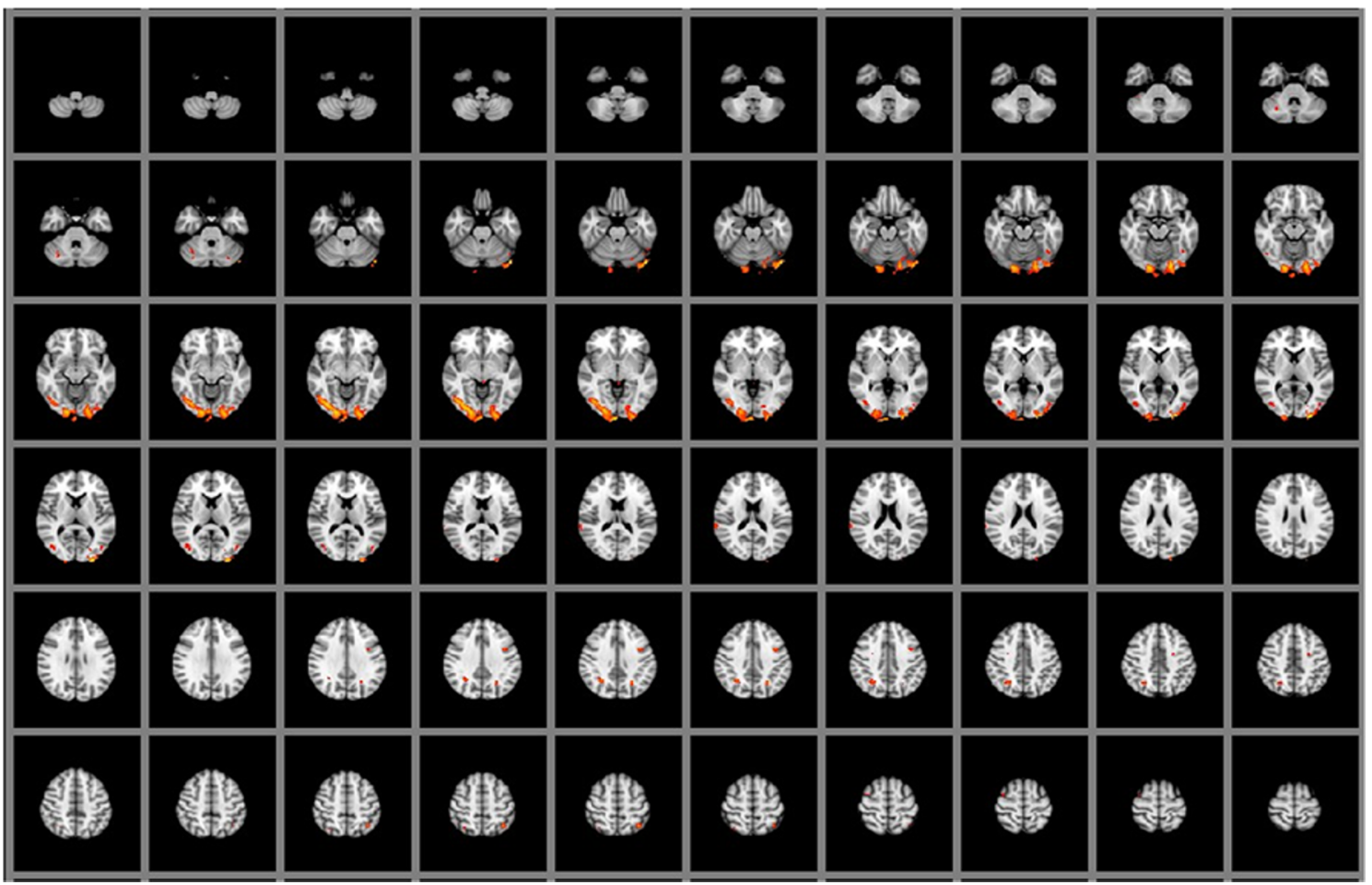

Fig. 1 Graphical presentation of the brain areas that were significantly stronger activated in laryngectomy patients than in healthy volunteers in response to task 3 
Table 4 Response to task 4

\begin{tabular}{|c|c|c|c|c|c|}
\hline Juelich histological atlas & $Z$ value & $P$ value & $\mathrm{x}$ & $\mathrm{y}$ & $\mathrm{z}$ \\
\hline \multicolumn{6}{|l|}{ Activation stronger in healthy volunteers than in laryngectomy patients } \\
\hline Left visual cortex V2 & 2.65 & $<0.0000$ & 52 & 15 & 33 \\
\hline Left visual cortex V1 & 2.53 & $<0.0000$ & 48 & 15 & 37 \\
\hline Right visual cortex V3 & 2.53 & $<0.0000$ & 30 & 22 & 32 \\
\hline Left Broca's area & 3.42 & 0.0035 & 68 & 67 & 47 \\
\hline Right inferior parietal lobule Pga & 3.41 & 0.0002 & 23 & 35 & 50 \\
\hline Right visual cortex V1 & 2.40 & $<0.0000$ & 35 & 26 & 38 \\
\hline Left inferior parietal lobule pgp & 2.39 & 0.0132 & 62 & 27 & 50 \\
\hline \multicolumn{6}{|c|}{ Activation stronger activated in laryngectomy patients than in healthy volunteers } \\
\hline Left visual cortex V2 & 2.56 & 0.0057 & 55 & 13 & 33 \\
\hline Left premotor cortex & 2.48 & 0.0028 & 57 & 56 & 69 \\
\hline Right secondary somatosensory cortex/parietal operculum OP1 & 2.42 & 0.0433 & 23 & 49 & 47 \\
\hline Left visual cortex V1 & 2.41 & 0.0008 & 46 & 18 & 37 \\
\hline Right visual cortex V2 & 2.39 & 0.0068 & 37 & 17 & 32 \\
\hline
\end{tabular}

dependent on the duration of rehabilitation and therefore might influence the results. This hypothesis still needs confirmation. On the other hand, the number of sessions to finish rehabilitation depended on individual abilities of patients. At inclusion to the study, all the patients had finished their speech rehabilitation with a positive outcome. Therefore, they were clinically diagnosed as positively rehabilitated. Considering this diagnosis, the group may be considered homogenous. Secondly, study participants were not selected according to education and profession, which also may have an impact on language processing. Thirdly, a more detailed analysis of inter-subject variability would be necessary to find other possible covariates of the outcome. For instance, differences in activations may be related to cognitive abilities of subjects, which are crucial to understand if changes in cortical activations are compensatory or maladaptive. As results of the current preliminary study appeared encouraging to us, we are now starting larger program, including cognitive and psychological testing. Therefore, further investigation is necessary involving more sizeable and carefully composed study groups to validate the current findings.

In conclusion, this study provides the first evidence of altered cortical activation in response to language tasks in patients after a laryngectomy compared with healthy volunteers, which may be considered brain plasticity in response to a laryngectomy.

Funding information This study was not supported by any funding.

\section{Compliance with ethical standards}

Conflict of interest The authors declare that they have no conflict of interest.
Ethical approval All procedures performed in studies involving human participants were in accordance with the ethical standards of the institutional and/or national research committee and with the 1964 Helsinki declaration and its later amendments or comparable ethical standards. The study was approved by the Institutional Review Board.

Informed consent Informed consent was obtained from all individual participants included in the study.

Open Access This article is licensed under a Creative Commons Attribution 4.0 International License, which permits use, sharing, adaptation, distribution and reproduction in any medium or format, as long as you give appropriate credit to the original author(s) and the source, provide a link to the Creative Commons licence, and indicate if changes were made. The images or other third party material in this article are included in the article's Creative Commons licence, unless indicated otherwise in a credit line to the material. If material is not included in the article's Creative Commons licence and your intended use is not permitted by statutory regulation or exceeds the permitted use, you will need to obtain permission directly from the copyright holder. To view a copy of this licence, visit http://creativecommons.org/licenses/by/4.0/.

\section{References}

1. Bogen JE, Bogen GM (1976) Wernicke's region-where is it? Ann N Y Acad Sci 280:834-843

2. Bramati IE, Rodrigues EC, Simões EL, Melo B, Höfle S, Moll J, Lent R, Tovar-Moll F (2019) Lower limb amputees undergo longdistance plasticity in sensorimotor functional connectivity. Sci Rep 9:2518

3. Hurren A, Miller N (2017) Voice outcomes post total laryngectomy. Curr Opin Otolaryngol Head Neck Surg 25:205-210

4. Fridriksson J, Fillmore P, Guo D, Rorden C (2015) Chronic Broca's aphasia is caused by damage to Broca's and Wernicke's areas. Cereb Cortex 25:4689-4696

5. Friedman L, Kenny JT, Wise AL, Wu D, Stuve TA, Miller DA, Jesberger JA, Lewin JS (1998) Brain activation during silent word generation evaluated with functional MRI. Brain Lang 64:231-256 
6. Guediche S, Holt LL, Laurent P, Lim SJ, Fiez JA (2015) Evidence for cerebellar contributions to adaptive plasticity in speech perception. Cereb Cortex 25:1867-1877

7. Hauck T, Probst M, Zimmer C, Ringel F, Meyer B, Wohlschlaeger A, Krieg SM (2018) Language function shows comparable cortical patterns by functional MRI and repetitive nTMS in healthy volunteers. Brain Imaging Behav 3:1071-1092

8. Krestel H (2013) Language and brain: historical introduction to models of language and aphasia. Swiss Arch Neurol Psychiatr Psychother 164:262-265

9. Jenkinson M, Smith SM (2001) A global optimisation method for robust affine registration of brain images. Med Image Anal 5: $143-156$

10. Woolrich MW, Ripley BD, Brady JM, Smith SM (2001) Temporal autocorrelation in univariate linear modelling of FMRI data. NeuroImage 14:1370-1386

11. Lorenz KJ (2017) Rehabilitation after total laryngectomy —a tribute to the pioneers of voice restoration in the last two centuries. Front Med 26:81

12. Zenga J, Goldsmith T, Bunting G, Deschler DG (2018) State of the art: rehabilitation of speech and swallowing after total laryngectomy. Oral Oncol 86:38-47

13. Mertl J, Žáčková E, Řepová B (2018) Quality of life of patients after total laryngectomy: the struggle against stigmatization and social exclusion using speech synthesis. Disabil Rehabil Assist Technol 13:342-352

14. van Sluis KE, van der Molen L, van Son RJJH, Bhairosing PA, van den Brekel MWM (2018) Objective and subjective voice outcomes after total laryngectomy: a systematic review. Eur Arch Otorhinolaryngol 275:11-26

15. Wierzchowska M, Burduk PK (2011) Early and late complications after implantation of the Provox 2 voice prosthesis in patients after total laryngectomy. Otolaryngol Pol 65:184-187

16. Bilewicz R, Burduk PK, Kopczynski A, Wierzchowska M (2007) The surgery voice rehabilitation after total laryngectomy with the Provox system. Otolaryngol Pol 61:265-270

17. Binder JR (2015) The Wernicke area: modern evidence and a reinterpretation. Neurology 85:2170-2175

18. Black DF, Vachha B, Mian A, Faro SH, Maheshwari M, Sair HI, Petrella JR, Pillai JJ, Welker K (2017) American Society of Functional Neuroradiology-recommended fMRI paradigm algorithms for presurgical language assessment. AJNR Am J Neuroradiol 38:E65-E73

19. Mahdavi A, Houshmand S, Oghabian MA, Zarei M, Mahdavi A, Shoar MH, Ghanaati H (2011) Developing optimized fMRI protocol for clinical use: comparison of different language paradigms. J Magn Reson Imaging 34:413-419

20. Wang Y, Holland SK, Vannest J (2012) Concordance of MEG and fMRI patterns in adolescents during verb generation. Brain Res 1447:79-90

21. Popp M, Trumpp NM, Kiefer M (2019) Brain activation during conceptual processing of action and sound verbs. Adv Cogn Psychol 15:236-255

22. Jenkinson M, Bannister P, Brady M, Smith S (2002) Improved optimization for the robust and accurate linear registration and motion correction of brain images. Neuroimagine 17:825-841

23. Worsley KJ (2012) Statistical analysis of activation images. Ch 14, in functional MRI: an introduction to methods. Oxford Scholarship Online

24. Amunts K, Kedo O, Kindler M, Pieperhoff P, Mohlberg H, Shah NJ, Habel U, Schneider F, Zilles K (2005) Cytoarchitectonic mapping of the human amygdala, hippocampal region and entorhinal cortex: intersubject variability and probability maps. Anat Embryol 210:343-352
25. Mesulam MM (1998) From sensation to cognition. Brain 121: 1013-1052

26. Nakai Y, Jeong JW, Brown EC, Rothermel R, Kojima K, Kambara T, Shah A, Mittal S, Sood S, Asano E (2017) Three- and fourdimensional mapping of speech and language in patients with epilepsy. Brain 140:1351-1370

27. Nathaniel-James DA, Fletcher P, Frith CD (1997) The functional anatomy of verbal initiation and suppression using the Hayling test. Neuropsychologia 35:559-566

28. Prpic N (2015) Language processing-role of inferior parietal lobule. Gyrus 3:173-175

29. Rapp AM, Mutschler DE, Erb M (2012) Where in the brain is nonliteral language? A coordinate-based meta-analysis of functional magnetic resonance imaging studies. Neuroimage 63:600-610

30. Ripamonti E, Frustaci M, Zonca G, Aggujaro S, Molteni F, Luzzatti C (2018) Disentangling phonological and articulatory processing: a neuroanatomical study in aphasia. Neuropsychologia 121:175-185

31. Sakai KL, Hashimoto R, Homae F (2001) Sentence processing in the cerebral cortex. Neurosci Res 39:1-10

32. Schubotz RI, von Cramon DY (2001) Functional organization of the lateral premotor cortex: fMRI reveals different regions activated by anticipation of object properties, location and speed. Brain Res Cogn Brain Res 11:97-112

33. Shibata M, Abe J, Terao A, Miyamoto T (2007) Neural mechanisms involved in the comprehension of metaphoric and literal sentences: an fMRI study. Brain Res 29:1166-1192

34. Taylor JS, Rastle K, Davis MH (2013) Can cognitive models explain brain activation during word and pseudoword reading? A meta-analysis of 36 neuroimaging studies. Psychol Bull 139: 766-791

35. Thioux M, Pesenti M, Costes N, De Volder A, Seron X (2005) Task-independent semantic activation for numbers and animals. Brain Res Cogn Brain Res 24:284-290

36. Wernicke K (1970) The aphasia symptom-complex: a psychological study on an anatomical basis. Arch Neurol 22:280-282

37. Whitney C, Weis S, Krings T, Huber W, Grossman M, Kircher T (2009) Task-dependent modulations of prefrontal and hippocampal activity during intrinsic word production. J Cogn Neurosci 21: 697-712

38. Wiese H, Stude P, Nebel K, de Greiff A, Forsting M, Diener HC, Keidel M (2004) Movement preparation in self-initiated versus externally triggered movements: an event-related fMRI-study. Neurosci Lett 371:220-225

39. Woodward TS, Ruff CC, Ngan ET (2006) Short- and long-term changes in anterior cingulate activation during resolution of taskset competition. Brain Res 1068:161-169

40. Worsley KJ (2001) Statistical analysis of activation images. In: Jezzard P, Matthews PM, Smith SM. (Eds.) Functional MRI: an introduction to methods. Oxford Scholarship Online

41. Wright A (2019) Higher cortical functions: language. Neuroscience Online. McGovern Medical School at UTHealth, Department of Neurobiology and Anatomy. Available via https://nba.uth.tmc. edu/neuroscience/m/s4/chapter08.html. Accessed 14 Sep 2019

42. Wu SJ, Jenkins LM, Apple AC, Petersen J, Xiao F, Wang L, Yang FG (2019) Longitudinal fMRI task reveals neural plasticity in default mode network with disrupted executive-default coupling and selective attention after traumatic brain injury. Brain Imaging Behav:1-13. https://doi.org/10.1007/s11682-019-00094-8

43. Yang J, Shu H (2016) Involvement of the motor system in comprehension of non-literal action language: a meta-analysis study. Brain Topogr 29:94-107

Publisher's note Springer Nature remains neutral with regard to jurisdictional claims in published maps and institutional affiliations. 